\title{
Moderate low temperature protects the ischemic brain injury by activating SIRT1 through inhibition of F0X01/PINK1/Parkin axis mediated mitophagy in a
} mouse

\section{Yaobin Zhu}

Cardiovascular Surgery $₫$,Beijing Children's Hospital, Capital Medical University, National Center for Children's Health

\section{Yaping Zhang}

The Heart Center, Beijing Friendship Hospital, Capital Medical University

\section{Nan Ding}

Cardiovascular Surgery $₫$,Beijing Children's Hospital, Capital Medical University, National Center for Children's Health

\section{Hanlu Yi}

Cardiovascular Surgery $\bigotimes, B e i j i n g$ Children's Hospital, Capital Medical University, National Center for Children's Health

\section{Xing Fan}

Pediatric Heart Center, Beijing Anzhen Hospital, Capital Medical University

\section{Zhiqiang Li ( $\nabla$ kjw12w@sohu.com )}

Beijing Children's Hospital

\section{Research}

Keywords: Moderate low temperature, ischemic brain injury, SIRT1, FOX01/PINK1/Parkin axis, mitophagy

Posted Date: February 25th, 2020

DOI: https://doi.org/10.21203/rs.2.24546/v1

License: (c) (i) This work is licensed under a Creative Commons Attribution 4.0 International License. Read Full License 


\section{Abstract}

Background: Several evidences suggested that the protective effect of hypothermia on brain injury is related to the inhibition of apoptosis and depends on the onset time of hypothermia and the degree of brain maturation. We performed many experiments aimed to comprehensively explore the biological functions of moderate low temperature protects the ischemic brain injury in a mouse and its underlying mechanism.

Methods: 12 normal healthy C57BL6 mouse were selected, and moderate low temperature model mouse were selected. The biological functions of moderate low temperature protect the ischemic brain injury in a mouse and its underlying mechanism were performed to explore.

Results: Based on our results, we found that moderate hypothermia brain protection could alleviate cerebral injury caused by ischemia reperfusion in mouse. Hypothermic brain protection reduced the level of oxidative stress induced by ischemia reperfusion in mouse. Meso-hypothermic cerebral protection could inhibit excessive mitochondrial autophagy induced by ischemia reperfusion in mouse. Medium-low temperature brain protection could activate SIRT1 and inhibit FOX01/PINK1/Parkin pathway. Activation of SIRT1 in the hypoxia/reoxygenation model of hippocampal neurons could inhibit autophagy and oxidative stress by inhibiting the FOX01/PINK1/Parkin pathway.

Conclusions : Moderate low temperature protects the ischemic brain injury by activating SIRT1 through inhibition of FOX01/PINK1/Parkin axis mediated mitophagy in a mouse.

\section{Highlights}

1. Moderate low temperature can protect the ischemic brain injury in a mouse.

2. Moderate low temperature protects the ischemic brain injury by activating SIRT1 in a mouse.

3. Moderate low temperature protects the ischemic brain injury by activating SIRT1 through inhibition of F0X01/PINK1/Parkin axis in a mouse.

4. Moderate low temperature protects the ischemic brain injury by activating SIRT1 through inhibition of FOX01/PINK1/Parkin axis mediated mitophagy in a mouse.

\section{Introduction}

Ischemic cerebrovascular disease is a serious threat to human health disease, mortality and morbidity are higher, with the speeding up of the social population aging speed, incidence is rising year by year [1]. Ischemic cerebrovascular disease is associated with cerebral artery stenosis and atherosclerosis. Percutaneous endovascular stenting and carotid stent implantation are often used to restore the blood flow at the ischemic site clinically [2]. In addition to brain tissue, ischemia reperfusion injury can also occur in the heart, kidney and other parts, seriously affecting the therapeutic effect of surgery, so reducing ischemia reperfusion injury is the current urgent treatment of ischemic cerebrovascular disease [3, 4]. 
The pathogenesis of ischemic cerebrovascular disease is complex, it is not clear, which is mainly related to the following factors: genetics susceptible factors, glycosylation end products accumulation, inflammatory response and inflammatory factors $[5,6]$. Studies have proved that in ischemic cerebrovascular disease, form and function of mitochondria [7]. Abnormalities, mitochondrial swelling and degeneration including spine fracture, disappearance, vacuolation and mitochondrial dynamics changes [7]. Therefore, many research centers at home and abroad are now carrying out studies on the role of mitochondria in the pathogenesis of ischemic cerebrovascular disease [8].

Autophagy refers to the process in which cells become autophagosomes by encapsulating aging organelles and abnormal proteins in the cell through the double-layer membrane structure, and then fuse with lysosomes to degrade their contents [9-11]. Under normal circumstances, cells through autophagy can not only form amino acids, nucleotides and other substances for energy circulation through degradation, but also clear damaged or aging organelles and their metabolites in the cytoplasm [12]. Autophagy is divided into non-selective autophagy and selective autophagy. Abnormal autophagy in the body may lead to the generation and development of renal disease [13].

Silencing information regulatory factor-related Sirts are $\mathrm{NAD}^{+}$dependent deacetylases, which plays a role in cell survival and apoptosis through deacetylation [14]. Sirt1 is a silencing information regulator 1 , which is present in most nuclei and tissues of the eye cytoplasmic expression in the cornea, iris, lens, and retina [15]. The occurrence and development of various disease are being explored, such as diabetic keratopathy, cataract, uveitis, neuropathy, and degenerative diseases of the retina [16]. It has been confirmed that Sirt1 expression is inhibited in the cornea of diabetic mice, while Sirt1 expression is overexpressed, and it significantly promoted the healing ability of corneal epithelium after injury $[17,18]$. Mitochondrial oxidative stress is reflected in the fact that Sirt1 can reduce the relevant acetyl in mitochondria [19]. The downstream target of Sirt1 is Parkin, a factor related to mitochondrial autophagy [20]. Parkin is E3 ubiquitin ligase, PINK1 is serine/threonine kinase, located in the upstream of Parkin [21]. The signaling pathway Sirt1/ sirt1-foxo1/pink1-parkin is involved in the regulation of mitochondrial autophagy [22]. According to the possible role of Sirt1 in mitochondrial autophagy and the F0X01/ pink1parkin pathway it regulates Sirt1 on the level of mitochondrial autophagy, combined with the characteristics of ischemic cerebrovascular disease [23], we hypothesized that Sirt1 has an effect on ischemic cerebrovascular disease has the therapeutic effect of promoting injury healing. To test this hypothesis, we applied in vitro fineness cell culture technique and establishment of ischemic cerebrovascular disease model in mouse to study the signal pathway Sirt1/FOX01/ pink1-parkin mediated mitochondrial autophagy in ischemic cerebrovascular disease. This result has important significance: on the one hand to improve the $d$ ischemic cerebrovascular disease pathogenesis; On the other hand, it provides a new intervention way for the treatment of ischemic cerebrovascular disease.

\section{Materials And Methods}

Animals 
Male C57BL/ 6 mouse were anesthetized by intraperitoneal injection of chloral hydrate $(353 \mathrm{mg} / \mathrm{kg})$ with using the doppler excitation photo flowmeter monitors cerebral blood flow in cerebral cortex supplied by middle cerebral artery (MCA). The head was fixed on the animal skull, at the position of $2 \mathrm{~mm}$ after the anterior halogen, $6 \mathrm{~mm}$ to the right of the midline. Short focal cerebral ischemia, cerebral artery occlusion (MCAO) induction. The specific procedure was as follows: the common carotid artery was exposed and separated on the supine position of mice, the distal end was ligated, the proximal end was clamped with hemostatic forceps, a small incision was made with microsurgical scissors, and a 6-0 nylon monofilament suture was inserted into the internal cervical movement pulse $10 \mathrm{~mm}$ to block MCA source. After $60 \mathrm{~min}$ of ischemia, the single thread was removed to complete the reperfusion. The body temperature of the animals was maintained at $37^{\circ} \mathrm{C}$ by a heating lamp (FHC, Bowdoinham) at the middle and $2 \mathrm{~h}$ after the start of reperfusion. At the beginning of reperfusion, 7 was injected into the lateral ventricle of mouse. In order to determine the volume of cerebral infarction, mouse was anesthetized $24 \mathrm{~h}$ after surgery, and their heads were severed TTC staining was performed on coronal sections. The calculation formula of infarct volume percentage was: (The unstemmed areas on the left - right unstemmed area)/left unstemmed area*100\%. The experiment was divided into three groups: control (sham operation), model group, medium-low temperature protection group [24].

In the present study, neurological function score and cerebral infarction volume measurement were used to detect ischemia reperfusion injury in mouse. The expressions of oxidative stress related factors (ROS, MDA, SOD, GSH-px) in mouse were detected. Western blot was used to detect the expression of autophagy marker Ic3-II/I in mouse of the three groups, and to observe and detect the autophagy status of mitochondria in brain tissues by electron microscopy. Mouse were treated with SIRT1 inhibitor (Sirtinol) and divided into control group (sham operation), model group, medium-low temperature protection group, medium-low temperature protection + Sirtinol. WB and PCR expression of SIRT1, FOXO1 and PINK1/Parkin related proteins, autophagy Ic3-II / proteins, oxidative stress and cerebral infarction volume in the brain tissues of mice in each group.

\section{Cell}

A hypoxia/reoxygenation model was established using mouse hippocampal neuron cell HT22 cell line to simulate ischemia reperfusion injury. Group 1) control; 2) model; 3) model +SIRT1; 4) model + SIRT1 + FOX01. Transfection of SIRT1 and FOXO1 and expression of SIRT1, FOXO1 and PINK1/Parkin related proteins were detected in each group. The expressions of oxidative stress related factors and autophagy Ic3-II/I protein in each group were detected.

\section{TTC staining}

In this study, TTC staining was used to measure the volume of cerebral infarction. $4 \%$ chloral hydrate anesthesia $(100 \mathrm{mg} / \mathrm{kg})$, quickly remove brain, remove the olfactory bulb and hindbrain, taking four starting frontopolar coronary slices, about $1.5 \mathrm{~mm}$ thick, immediately put $2 \%$ TTC solution $(0.01 \mathrm{M}$ phosphate buffer preparation $\mathrm{pH} 7.4$ slightly), $37^{\circ} \mathrm{C}$ avoid light incubation for $30 \mathrm{~min}$. Then soak and fix 
with $10 \%$ paraformaldehyde solution. Infarcted areas appear white and non-infarcted areas appear red. Champion Image hpias-1000 high-resolution color pathological Image and text report analysis system was applied to measure the area of cerebral infarction in each area, and the infarct volume was calculated according to the formula $V=t\left(A_{1}+\ldots A_{n}\right)-\left(A_{1}+A_{n}\right) t / 2$. Where $t$ is the slice thickness and $A$ is the infarct area [25].

\section{Expression detection of oxidative stress related factors}

Levels of oxidative stress related factors were measured using ELISA kit (Cyman system) according to the instruction manual.

\section{Western blotting}

Samples were prepared by lysis with proteinase inhibitor. The protein was quantified by BCA kit (Pierce Biotechnology, IL). Each sample were separated by $15 \%$ SDS-PAGE, and the membranes were incubated for $12 \mathrm{~h}$ at $4^{\circ} \mathrm{C}$ with antibodies, and then incubated with goat-anti-mouse IgG (Santa Cruz Biotechnology USA). The signals were detected with ECL reagents.

\section{Real-time reverse transcription-polymerase chain reaction}

Total RNA was extracted using TRIzol reagent (Invitrogen Life Technologies, Carlsbad, CA, USA) according to the manufacturer's instructions. The concentration of RNA was measured using a NanoDrop-1000 (Thermo Fisher Scientific, Waltham, MA, USA), and RNA integrity was determined by $1.5 \%$ agarose gel electrophoresis. cDNA synthesis was performed according to the manufacturer's recommendation. Briefly, RNA was reverse-transcribed (RT) using a ReverAid First Strand cDNA kit (Thermo Fisher Scientific). Under our experimental conditions, we used $\beta$-actin as an internal control for normalizing the expression levels of mRNA. cDNA products were then diluted at 20X, and the 10- $\mu$ I PCR mixture contained $1 \mu$ l diluted RT product, $5 \mu$ I SYBR-Green Master Mix, $2 \mu$ l RNase-free water, $1 \mu \mathrm{l}$ forward, and $1 \mu \mathrm{l}$ reverse primers. The reaction was incubated at $95^{\circ} \mathrm{C}$ for $10 \mathrm{~min}$ followed by 40 cycles of $95^{\circ} \mathrm{C}$ for $15 \mathrm{sec}$ and $65^{\circ} \mathrm{C}$ for $60 \mathrm{sec}$. The relative mRNAs expression was calculated using the $2^{-\Delta \Delta \mathrm{Ct}}$ method.

\section{Electron microscopy observation}

Placed the paraffin tissue section in room temperature $20 \mathrm{~min}$, the tissue sections were soaked in xylene for $10 \mathrm{~min}$, and then soaked for $10 \mathrm{~min}$ after replacing xylene, soak in anhydrous ethanol for $5 \mathrm{~min}$, immerse in $95 \%$ ethanol for $5 \mathrm{~min}$, soak in $70 \%$ ethanol for $5 \mathrm{~min}$. Boiling hot fix, electric or water bath pot heating $0.01 \mathrm{M}$ sodium citrate buffer (pH6.0), in a tissue section heating 10-15 min. Transparent at room temperature for $15 \mathrm{~min}$ in transparent solution. The slices were placed in the sealing solution, sealed at room temperature and away from light for $30 \mathrm{~min}$, and the excess liquid was thrown away. Observed the results under the electron microscopy and take photos. 
mimics and negative control oligonucleotides were synthesized by GenePharma Co., Ltd. (Shanghai, China). Cell transfection was performed using Lipofectamine 2000 reagents (Invitrogen Life Technologies) according to the manufacturer's protocol. Twenty-four hours after transfection, cells were collected for RT-PCR, Western blot analysis and functional assay.

\section{Statistical analysis}

Results were performed using SPSS 19.0, and presented as the mean \pm SD from three independent experiments. A value of $\mathrm{P}<0.05$ was considered to indicate a statistically significant difference.

\section{Results}

Moderate hypothermia brain protection could alleviate cerebral injury caused by ischemia reperfusion in mouse

Neurological function score and cerebral infarction volume measurement were used to detect ischemia reperfusion injury in mouse from control (sham operation), model group and medium-low temperature protection group (Fig. 1). Moderate hypothermia brain protection could alleviate cerebral injury caused by ischemia reperfusion in mouse.

Hypothermic brain protection reduced the level of oxidative stress induced by ischemia reperfusion in mouse

The expressions of oxidative stress related factors (ROS, MDA, SOD, GSH-px) in mouse from control (sham operation), model group and medium-low temperature protection group were detected (Fig.2). Hypothermic brain protection reduced the level of oxidative stress induced by ischemia reperfusion in mouse.

Meso-hypothermic cerebral protection could inhibit excessive mitochondrial autophagy induced by ischemia reperfusion in mouse

Western blot was used to detect the expression of autophagy marker Ic3-II / in mouse of the three groups, and to observe and detect the autophagy status of mitochondria in brain tissues by electron microscopy (Fig.3). Meso-hypothermic cerebral protection could inhibit excessive mitochondrial autophagy induced by ischemia reperfusion in mouse.

Medium-low temperature brain protection could activate SIRT1 and inhibit FOXO1/PINK1/Parkin pathway

The expression of SIRT1, FOXO1 and PINK1/Parkin related proteins, autophagy Ic3-II /I proteins, oxidative stress and cerebral infarction volume in the brain tissues of mouse from control (sham operation), model group, medium-low temperature protection group, medium-low temperature protection + Sirtinol were detected (Fig.4). Based on the results, medium-low temperature brain protection could activate SIRT1 and inhibit FOXO1/PINK1/Parkin pathway 
Activation of SIRT1 in the hypoxia/reoxygenation model of hippocampal neurons could inhibit autophagy and oxidative stress by inhibiting the FOXO1/PINK1/Parkin pathway

Transfection of SIRT1 and FOXO1 and expression of SIRT1, FOXO1 and PINK1/Parkin related proteins were detected in each group (Fig.5). The expressions of oxidative stress related factors and autophagy Ic3-ii /I protein in each group were detected (Fig.5). Activation of SIRT1 in the hypoxia/reoxygenation model of hippocampal neurons could inhibit autophagy and oxidative stress by inhibiting the FOX01/PINK1/Parkin pathway.

\section{Discussion}

Ischemic cerebrovascular disease is a serious threat with the speeding up of the social population aging speed. Ischemic cerebrovascular disease is associated with cerebral artery stenosis and atherosclerosis [1-4]. Ischemia reperfusion injury can also occur in the heart, kidney and other parts, seriously affecting the therapeutic effect of surgery. The pathogenesis of ischemic cerebrovascular disease is complex, which is mainly related to the following factors: genetics susceptible factors, glycosylation end products accumulation, inflammatory response and inflammatory factors [2-9]. Many research centers at home and abroad are now carrying out studies on the role of mitochondria in the pathogenesis of ischemic cerebrovascular disease [11-15].

Abnormal autophagy in the body may lead to the generation and development of renal disease. Currently, studies have found that cisplatin induced acute kidney injury and renal ischemia in the model. In the reperfusion injury model, autophagy is enhanced, which plays a role in kidney protection. Autophagy has also been shown to be associated with kidney aging. Sirts play a role in cell survival and apoptosis through deacetylation. Sirt1 is present in most nuclei and tissues of the eye cytoplasmic expression in the cornea, iris, lens, and retina [26].

The signaling pathway Sirt1/ sirt1-foxo1/pink1-parkin is involved in the regulation of mitochondrial autophagy. According to the possible role of Sirt1 in mitochondrial autophagy and the FOX01/ pink1parkin pathway it regulates Sirt1 on the level of mitochondrial autophagy. In this study, we applied in vitro fineness cell culture technique and establishment of ischemic cerebrovascular disease model in mouse to study the signal pathway Sirt1/FOX01/ pink1-parkin mediated mitochondrial autophagy in ischemic cerebrovascular disease [27-30]. Based on our results, we found that moderate hypothermia brain protection could alleviate cerebral injury caused by ischemia reperfusion in mouse. Hypothermic brain protection reduced the level of oxidative stress induced by ischemia reperfusion in mouse. Mesohypothermic cerebral protection could inhibit excessive mitochondrial autophagy induced by ischemia reperfusion in mouse. Medium-low temperature brain protection could activate SIRT1 and inhibit F0X01/PINK1/Parkin pathway. Activation of SIRT1 in the hypoxia/reoxygenation model of hippocampal neurons could inhibit autophagy and oxidative stress by inhibiting the F0X01/PINK1/Parkin pathway.

\section{Conclusion}


In summary, moderate low temperature protects the ischemic brain injury by activating SIRT1 through inhibition of FOX01/PINK1/Parkin axis mediated mitophagy in a mouse. These results have important significance: on the one hand to improve the $d$ ischemic cerebrovascular disease pathogenesis; On the other hand, it provides a new intervention way for the treatment of ischemic cerebrovascular disease.

\section{Declarations}

\section{Abbreviation}

Not Applicable.

\section{Ethics approval and consent to participate}

Ethical approval was provided by the Medical Ethics Committee of Beijing Children's Hospital, Capital Medical University, National Center for Children's Health.

\section{Consent for publication}

All of the authors have Consented to publish this research.

\section{Availability of data and material}

All data generated or analyzed during this study are included in this published article.

\section{Competing interests}

The authors declare no conflict of interest.

\section{Funding}

This research was sponsored by the Medical and Health Technology Project.

This study is supported by Beijing Natural Science Foundation (NO. 7184204, NO. 7182042), Beijing Municipal Health Bureau High-Level Talent Cultivation (NO.2014-3-043, NO.2015-3-048, and NO.2015-3051), and Beijing Municipal Administration of Hospital Incubating Program (PX20166046), Research on the application of clinical characteristics of the Beijing Municipal Science and Technology Commission(Z171100001017048).

\section{Authors' contributions}

Each author has made an important scientific contribution to the study and has assisted with the drafting or revising of the manuscript.

\section{Acknowledgements}

Thanks for funding and the cooperation of the teamwork. 


\section{References}

1. Dawson, T.M., Ko, H.S. and Dawson, V.L. (2010) Genetic animal models of Parkinson's disease. Neuron, 66, 646-661.

2. Lu, B. (2009) Mitochondrial dynamics and neurodegeneration. Curr. Neurol. Neurosci. Rep., 9, 212219.

3. Clark, I.E., Dodson, M.W., Jiang, C., Cao, J.H., Huh, J.R., Seol, J.H., Yoo, S.J., Hay, B.A. and Guo, M. (2006) Drosophila pink1 is required for mitochondrial function and interacts genetically with parkin. Nature, 441, 1162-1166.

4. Park, J., Lee, S.B., Lee, S., Kim, Y., Song, S., Kim, S., Bae, E., Kim, J., Shong, M., Kim, J.M. et al. (2006) Mitochondrial dysfunction in Drosophila PINK1 mutants is complemented by parkin. Nature, 441, 1157-1161.

5. Yang, Y., Gehrke, S., Imai, Y., Huang, Z., Ouyang, Y., Wang, J.W., Yang, L., Beal, M.F., Vogel, H. and Lu, B. (2006) Mitochondrial pathology and muscle and dopaminergic neuron degeneration caused by inactivation of Drosophila Pink1 is rescued by Parkin. Proc. Natl Acad. Sci. USA, 103, 10793-10798.

6. Yang, Y., Ouyang, Y., Yang, L., Beal, M.F., McQuibban, A., Vogel, H. and Lu, B. (2008) Pink1 regulates mitochondrial dynamics through interaction with the fission/fusion machinery. Proc. Natl Acad. Sci. USA, 105, 7070-7075.

7. Deng, H., Dodson, M.W., Huang, H. and Guo, M. (2008) The Parkinson's disease genes pink1 and parkin promote mitochondrial fission and/or inhibit fusion in Drosophila. Proc. Natl Acad. Sci. USA, $105,14503-14508$.

8. Park, J., Lee, G. and Chung, J. (2009) The PINK1-Parkin pathway is involved in the regulation of mitochondrial remodeling process. Biochem. Biophys. Res. Commun., 378, 518-523.

9. Poole, A.C., Thomas, R.E., Andrews, L.A., McBride, H.M., Whitworth, A.J. and Pallanck, L.J. (2008) The PINK1/Parkin pathway regulates mitochondrial morphology. Proc. Natl Acad. Sci. USA, 105, 16381643.

10. Exner, N., Treske, B., Paquet, D., Holmstrom, K., Schiesling, C., Gispert, S., Carballo-Carbajal, I., Berg, D., Hoepken, H.H., Gasser, T. et al. (2007) Loss-of-function of human PINK1 results in mitochondrial pathology and can be rescued by parkin. J. Neurosci., 27, 12413-12418.

11. Dagda, R.K., Cherra, S.J. III, Kulich, S.M., Tandon, A., Park, D. and Chu, C.T. (2009) Loss of PINK1 function promotes mitophagy through effects on oxidative stress and mitochondrial fission. J. Biol. Chem., 284, 13843-13855.

12. Lutz, A.K., Exner, N., Fett, M.E., Schlehe, J.S., Kloos, K., Lammermann, K., Brunner, B., Kurz-Drexler, A., Vogel, F., Reichert, A.S. et al. (2009) Loss of parkin or PINK1 function increases Drp1-dependent mitochondrial fragmentation. J. Biol. Chem., 284, 22938-22951.

13. Sandebring, A., Thomas, K.J., Beilina, A., van der Brug, M., Cleland, M.M., Ahmad, R., Miller, D.W., Zambrano, I., Cowburn, R.F., Behbahani, H. et al. (2009) Mitochondrial alterations in PINK1 deficient 
cells are influenced by calcineurin-dependent dephosphorylation of dynamin-related protein 1. PLoS One, 4, e5701.

14. Mortiboys, H., Thomas, K.J., Koopman, W.J., Klaffke, S., Abou-Sleiman, P., Olpin, S., Wood, N.W., Willems, P.H., Smeitink, J.A., Cookson, M.R. et al. (2008) Mitochondrial function and morphology are impaired in parkin-mutant fibroblasts. Ann. Neurol., 64, 555-565.

15. Hatano, Y., Li, Y., Sato, K., Asakawa, S., Yamamura, Y., Tomiyama, H., Yoshino, H., Asahina, M., Kobayashi, S., Hassin-Baer, S. et al. (2004) Novel PINK1 mutations in early-onset parkinsonism. Ann. Neurol., 56, 424-427.

16. Liu, W., Vives-Bauza, C., Acin-Perez, R., Yamamoto, A., Tan, Y., Li, Y., Magrane, J., Stavarache, M.A., Shaffer, S., Chang, S. et al. (2009) PINK1 defect causes mitochondrial dysfunction, proteasomal deficit andalpha-synuclein aggregation in cell culture models of Parkinson's disease. PLoS One, 4, e4597.

17. Schlehe, J.S., Lutz, A.K., Pilsl, A., Lammermann, K., Grgur, K., Henn, I.H., Tatzelt, J. and Winklhofer, K.F. (2008) Aberrant folding of pathogenic Parkin mutants: aggregation versus degradation. J. Biol. Chem., 283, 13771-13779.

18. Smirnova, E., Shurland, D.L., Ryazantsev, S.N. and van der Bliek, A.M. (1998) A human dynaminrelated protein controls the distribution of mitochondria. J. Cell Biol., 143, 351-358.

19. Delettre, C., Lenaers, G., Griffoin, J.M., Gigarel, N., Lorenzo, C., Belenguer, P., Pelloquin, L., Grosgeorge, J., Turc-Carel, C., Perret, E. et al. (2000) Nuclear gene OPA1, encoding a mitochondrial dynaminrelated protein, is mutated in dominant optic atrophy. Nat. Genet., 26, 207-210.

20. Santel, A. and Fuller, M.T. (2001) Control of mitochondrial morphology by a human mitofusin. J. Cell Sci., 114, 867-874.

21. Eura, Y., Ishihara, N., Yokota, S. and Mihara, K. (2003) Two mitofusin proteins, mammalian homologues of FZO, with distinct functions are both required for mitochondrial fusion. J. Biochem., 134, 333-344.

22. Santel, A., Frank, S., Gaume, B., Herrler, M., Youle, R.J. and Fuller, M.T. (2003) Mitofusin-1 protein is a generally expressed mediator of mitochondrial fusion in mammalian cells. J. Cell Sci., 116, 27632774.

23. Li, Z., Okamoto, K., Hayashi, Y. and Sheng, M. (2004) The importance of dendritic mitochondria in the morphogenesis and plasticity of spines and synapses. Cell, 119, 873-887.

24. Ziviani, E., Tao, R.N. and Whitworth, A.J. (2010) Drosophila parkin requires PINK1 for mitochondrial translocation and ubiquitinates mitofusin. Proc. Natl Acad. Sci. USA, 107, 5018-5023.

25. Gegg, M.E., Cooper, J.M., Chau, K.Y., Rojo, M., Schapira, A.H. and Taanman, J.W. (2010) Mitofusin 1 and mitofusin 2 are ubiquitinated in a PINK1/parkin-dependent manner upon induction of mitophagy. Hum. Mol. Gen., 19, 4861-4870.

26. Margineantu, D.H., Gregory Cox, W., Sundell, L., Sherwood, S.W., Beechem, J.M. and Capaldi, R.A. (2002) Cell cycle dependent morphology changes and associated mitochondrial DNA redistribution in mitochondria of human cell lines. Mitochondrion, 1, 425-435. 
27. Mitra, K., Wunder, C., Roysam, B., Lin, G. and Lippincott-Schwartz, J. (2009) A hyperfused mitochondrial state achieved at G1-S regulates cyclin E buildup and entry into S phase. Proc. Natl Acad. Sci. USA, 106, 11960-11965.

28. Taguchi, N., Ishihara, N., Jofuku, A., Oka, T. and Mihara, K. (2007) Mitotic phosphorylation of dynamin-related GTPase Drp1 participates in mitochondrial fission. J. Biol. Chem., 282, 1152111529.

29. Cipolat, S., Martins de Brito, O., Dal Zilio, B. and Scorrano, L. (2004) OPA1 requires mitofusin 1 to promote mitochondrial fusion. Proc. Natl Acad. Sci. USA, 101, 15927-15932.

30. Griparic, L., van der Wel, N.N., Orozco, I.J., Peters, P.J. and van der Bliek, A.M. (2004) Loss of the intermembrane space protein Mgm1/OPA1 induces swelling and localized constrictions along the lengths of mitochondria. J. Biol. Chem., 279, 18792-18798.

\section{Figures}

A

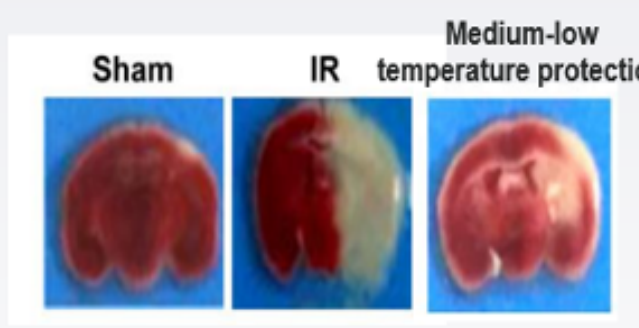

\section{B}

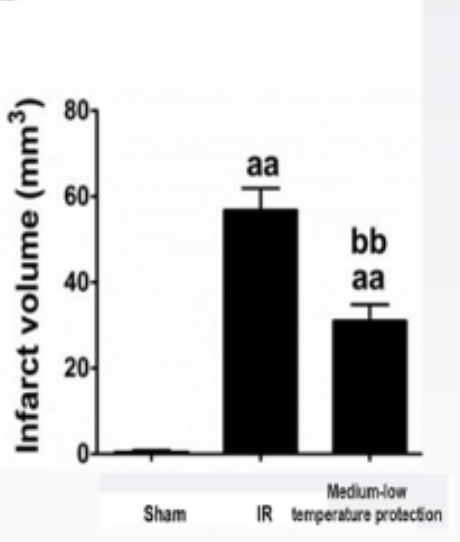

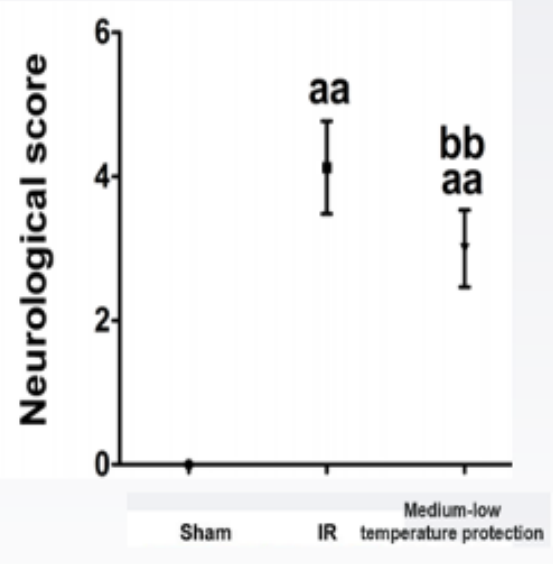

\section{Figure 2}

Moderate hypothermia brain protection could alleviate cerebral injury caused by ischemia reperfusion in mouse 

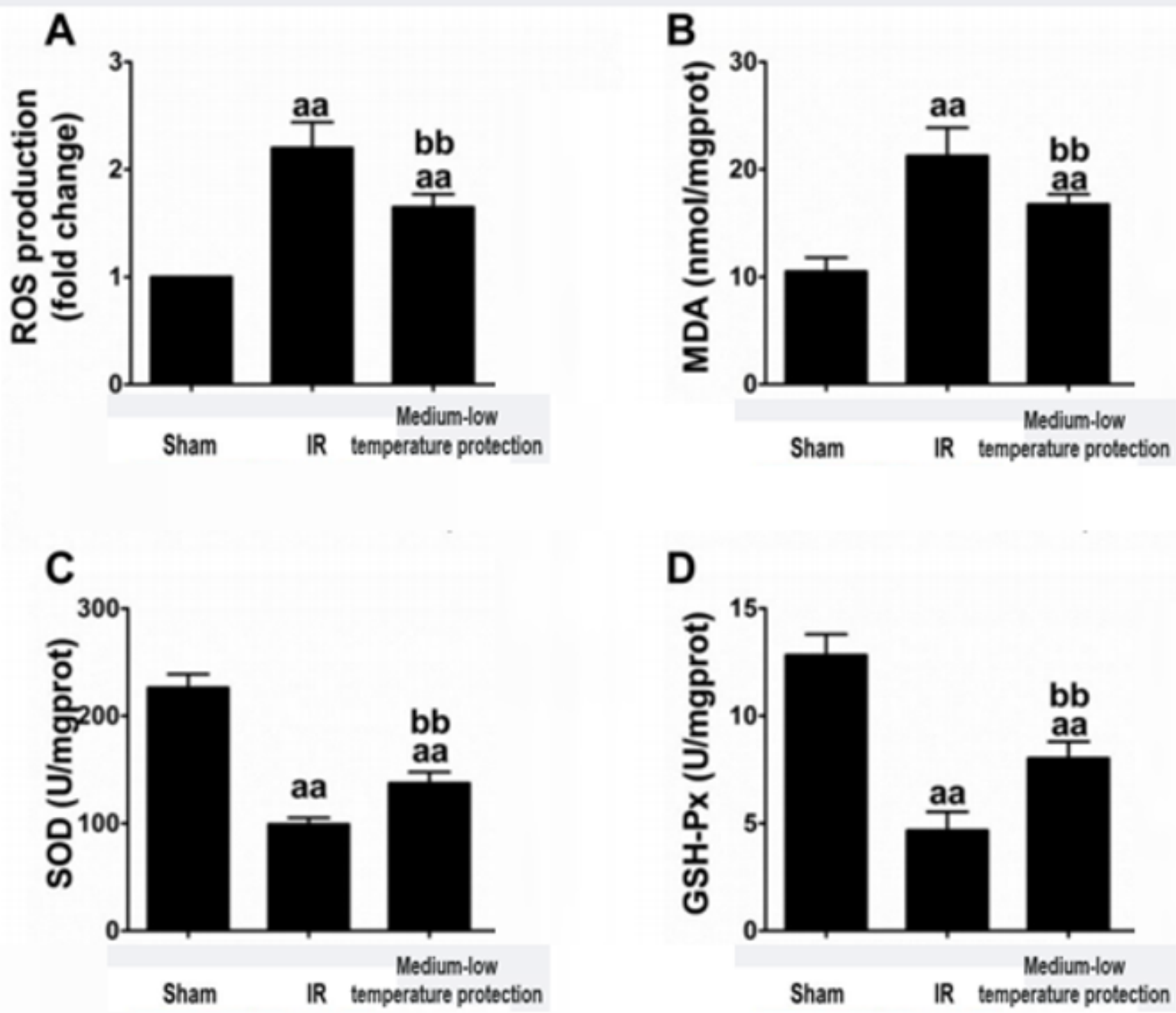

Figure 4

Hypothermic brain protection reduced the level of oxidative stress induced by ischemia reperfusion in mouse 


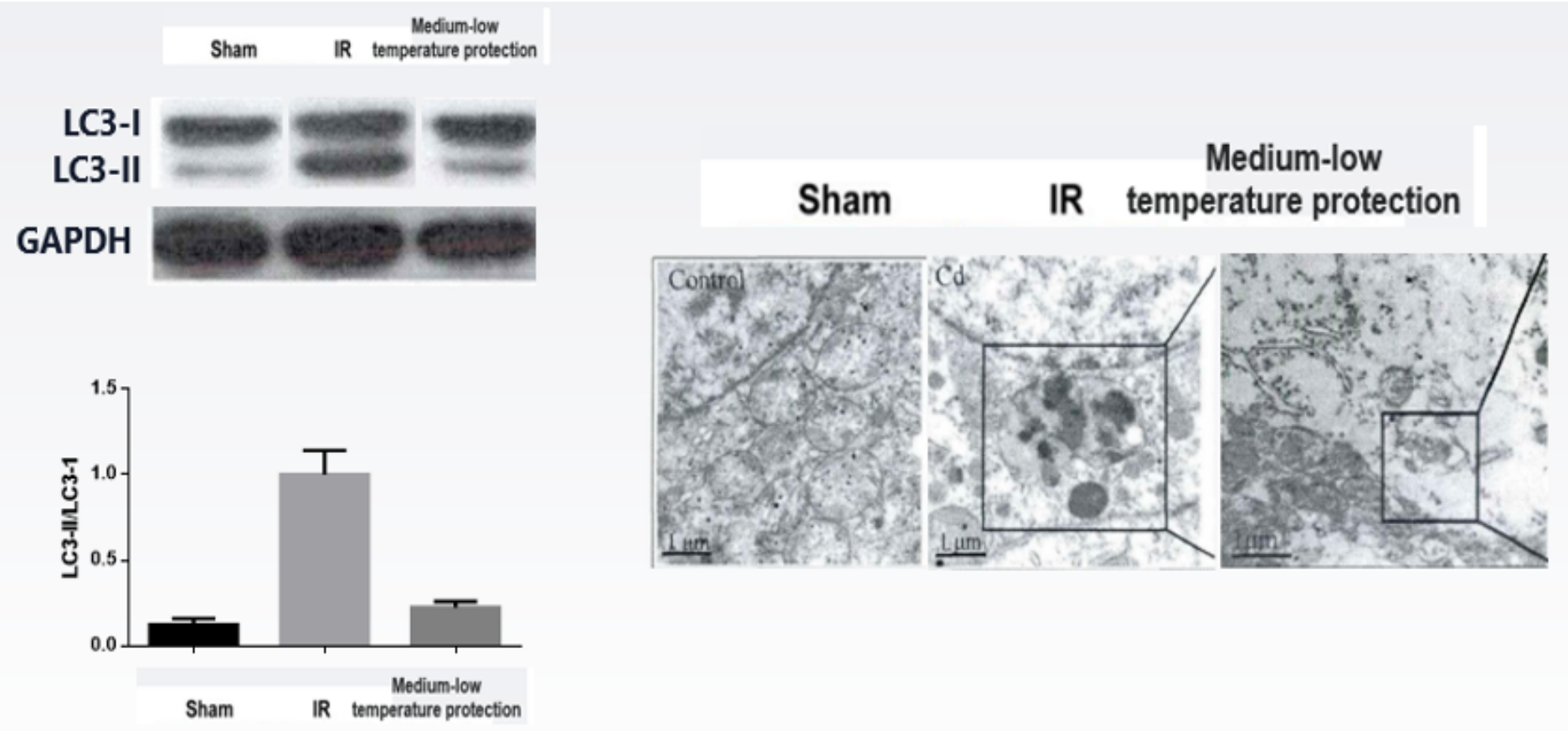

Figure 5

Meso-hypothermic cerebral protection could inhibit excessive mitochondrial autophagy induced by ischemia reperfusion in mouse

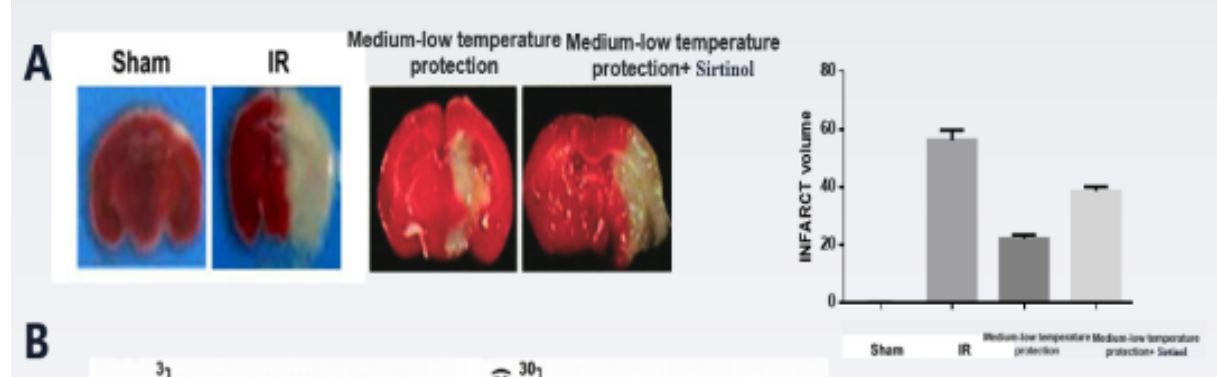

C
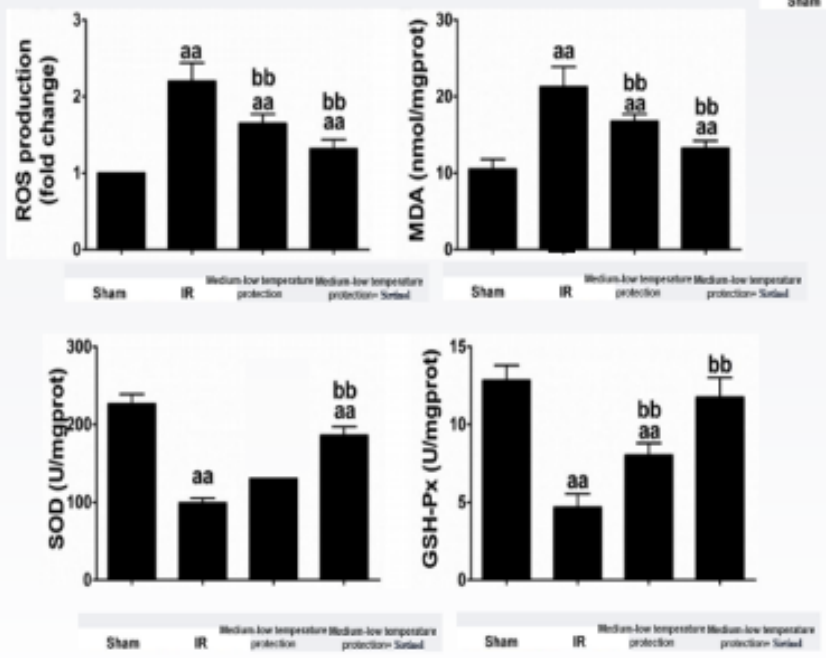

D

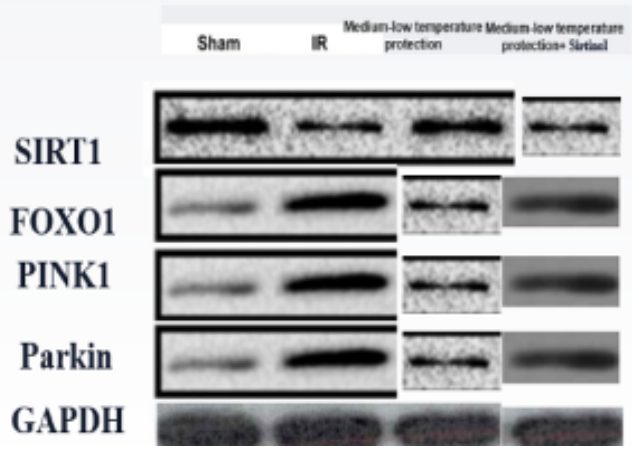

Figure 7

Medium-low temperature brain protection could activate SIRT1 and inhibit F0X01/PINK1/Parkin pathway 
A

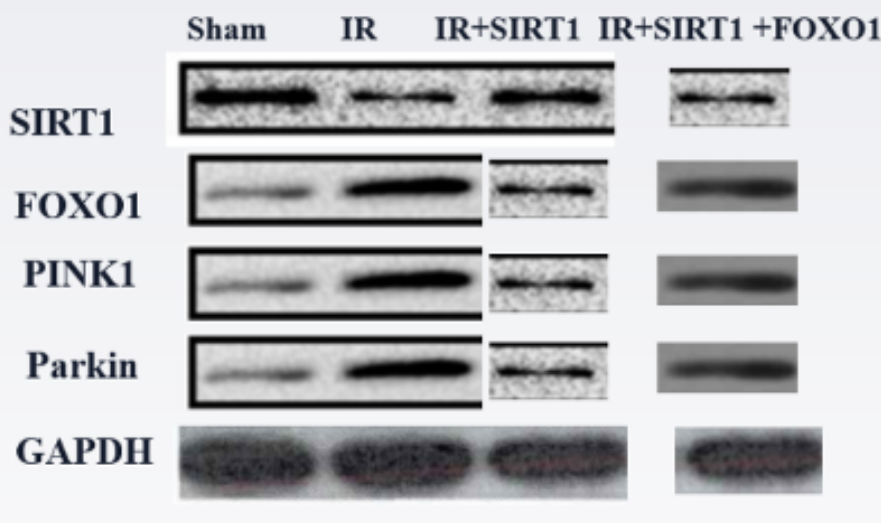

B

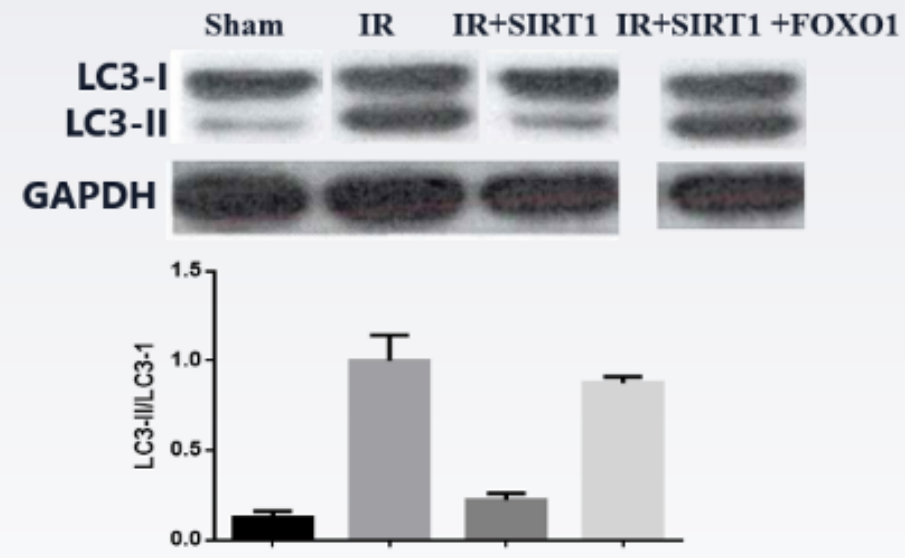

Sham IR IR+SIRT1 IR+SIRT1 +FOXO1

C
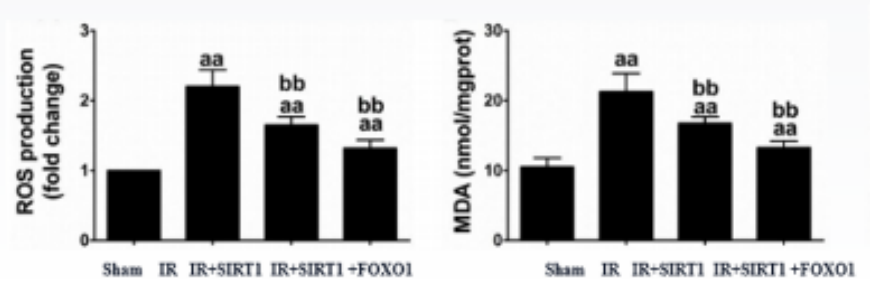

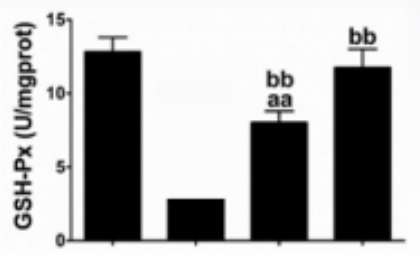

Sham IR IR+5IRTI IR+SIRTI+FOXO1

Figure 10

Activation of SIRT1 in the hypoxia/reoxygenation model of hippocampal neurons could inhibit autophagy and oxidative stress by inhibiting the FOX01/PINK1/Parkin pathway 